\title{
Philosophiques
}

\section{Au fil conducteur du scepticisme : science et métaphysique chez Glanvill}

\section{Frédéric Brahami}

Volume 35, numéro 1, printemps 2008

Les valeurs de l'ironie (1) et le scepticisme à l'âge classique (2)

URI : https://id.erudit.org/iderudit/018246ar

DOI : https://doi.org/10.7202/018246ar

Aller au sommaire du numéro

\section{Éditeur(s)}

Société de philosophie du Québec

ISSN

0316-2923 (imprimé)

1492-1391 (numérique)

Découvrir la revue

Citer cet article

Brahami, F. (2008). Au fil conducteur du scepticisme : science et métaphysique chez Glanvill. Philosophiques, 35(1), 207-222. https://doi.org/10.7202/018246ar

\section{Résumé de l'article}

Membre de la Royal Society, Joseph Glanvill a écrit une oeuvre complexe où le scepticisme le plus radical va de pair avec la plus grande confiance dans les progrès du savoir. Ce paradoxe apparent exprime une nouvelle conception de la science, résolument déprise de toute prétention à la saisie intuitive de la nature des choses. Le scepticisme devient alors non seulement la condition des progrès scientifiques, mais la méthode même de la science. Dans cette mesure, Glanvill tient une place importante, quoique discrète, dans l'histoire moderne de la philosophie critique. La distinction qu'il opère, pour répondre à l'argument cartésien du Dieu trompeur, entre la « certitude infaillible » et la " certitude indubitable ", marque en effet le moment où la science, consciente de ses règles, se sait elle-même relative. 


\title{
Au fil conducteur du scepticisme: science et métaphysique chez Glanvill
}

\author{
FRÉDÉRIC BRAHAMI \\ Université de Franche-Comté \\ fbrahami@wanadoo.fr
}

\begin{abstract}
RÉSUMÉ. - Membre de la Royal Society, Joseph Glanvill a écrit une œuvre complexe où le scepticisme le plus radical va de pair avec la plus grande confiance dans les progrès du savoir. Ce paradoxe apparent exprime une nouvelle conception de la science, résolument déprise de toute prétention à la saisie intuitive de la nature des choses. Le scepticisme devient alors non seulement la condition des progrès scientifiques, mais la méthode même de la science. Dans cette mesure, Glanvill tient une place importante, quoique discrète, dans l'histoire moderne de la philosophie critique. La distinction qu'il opère, pour répondre à l'argument cartésien du Dieu trompeur, entre la «certitude infaillible» et la «certitude indubitable», marque en effet le moment où la science, consciente de ses règles, se sait elle-même relative.
\end{abstract}

\begin{abstract}
The works of Joseph Glanvill, who was a fellow of the Royal Society, are complex: indeed, the most radical scepticism can be found to go hand in hand with the deepest trust in the advancement of knowledge. This apparent paradox bespeaks a new conception of science : a science that is definitely free from any claim to an intuitive comprehension of the nature of things. Scepticism thus becomes the condition of scientific progress as well as the very method of science itself. In so far forth, Glanvill has an important place, however unconspicuous, in the modern history of critical philosophy. In his answer to Descartes's argument of the Deus deceptor (a deceptive god), Glanvill makes a distinction between "infallible certainty" and "indubitable certainty", which indeed marks the moment when science, aware of its own rules, acknowledges its relativity.
\end{abstract}

Le scepticisme de Joseph Glanvill témoigne d'une mutation décisive dans le rapport que la science entretient à la vérité. Pour être radicale en effet, la critique des facultés que Glanvill mène tout au long de son œuvre n'aboutit pourtant jamais à désespérer de la science, mais se présente au contraire comme la condition même de son développement. Tel est le sens du titre volontairement paradoxal de l'ouvrage de 1665 : Scepsis Scientifica, or confest ignorance, the way to science. Le scepticisme, entendu d'abord comme l'aveu de l'ignorance, constitue en tant que tel la méthode pour parvenir à la science. Il semble que Glanvill se contente ainsi de mettre ses pas dans ceux de Bacon et de Descartes qui avaient, chacun à sa manière, fait précéder d'une pars destruens la pars construens de leurs systèmes. La théorie des idoles chez Bacon, le doute radical chez Descartes leur permettaient de faire table rase des savoirs figés en autorité, de manière à fonder la science sur le rapport immédiat de l'esprit à son objet. Mais Glanvill ne s'inscrit pas véritablement dans cette lignée, car chez lui le scepticisme n'est pas seulement un préalable méthodologique; on 
pourrait dire que c'est la manière d'être de la science. Or, pour que le scepticisme ne soit plus l'autre de la science mais sa condition, sa méthode et sa manière d'être, il faut que la science signifie autre chose que ce qu'à ses yeux elle signifiait jusque là. Si la science doit elle-même être sceptique, c'est qu'elle n'a plus pour but la vérité, comprise classiquement comme l'adéquation de l'esprit à la chose. Le scepticisme de Glanvill ne se réduit pas à la mise en œuvre d'une pars destruens préalable à la construction positive du savoir parce que son analyse des facultés de l'esprit le conduit à affirmer que nous ne pourrons jamais dépasser le stade des hypothèses : «the best principles [...] are but hypotheses ${ }^{1} »$. Que les meilleurs principes ne soient jamais que des hypothèses, voilà bien un énoncé radicalement sceptique, en ce qu'il signifie que la science ne rejoint jamais le réel, qu'elle ne peut jamais s'assurer que ses résultats sont adéquats à ce qui est. Aussi, l'abîme est-il infranchissable qui sépare l'ordre du discours de l'ordre de l'être.

La raison pour laquelle nous ne pouvons dépasser la formulation de principes hypothétiques tient au fait qu'il nous est absolument impossible de saisir la nécessité des réalités d'expérience: "Nous pouvons affirmer que les choses sont telles et telles, selon les principes que nous avons épousés, mais nous nous oublions étrangement lorsque nous défendons l'idée qu'il est nécessaire qu'elles soient telles dans la Nature, et qu'il est impossible qu'elles soient autrement ${ }^{2}$. " Encore Glanvill va-t-il plus loin : il ne se contente pas d'affirmer que nous devons faire notre deuil de l'absolu, il affirme que ce n'est que par ce deuil que nous pourrons faire progresser la science. Ainsi, ce n'est pas tant malgré son renoncement à l'absolu que la science est possible que grâce à lui qu'elle devient effective : la science n'est telle que d'assumer son caractère définitivement hypothétique, de se savoir elle-même relative à des facultés cognitives dont on sait que leur nature leur interdit définitivement toute appréhension intuitive du réel. La vérité prend alors un nouveau sens : elle ne dit pas ce qui est, pas plus qu'elle ne le reflète; elle construit une représentation de la nature dont elle sait qu'elle est un produit de l'esprit. Pour que naisse la science, il faut qu'elle sache que le réel l'excède et la déborde de toutes parts. C'est ainsi seulement qu'elle peut échapper au piège des analogies et des anticipations contre lesquelles déjà Bacon avait mis en garde ses petits-neveux ${ }^{3}$. S'il devait y avoir une intuition métaphysique de Glanvill ${ }^{4}$, ce serait sans doute cette claire conscience d'un abîme infranchissable entre le réel et l'appréhension que nous pouvons en avoir.

1. Scepsis Scientifica, dans Glanvill (1970), p. 145.

2. Ibid.

3. Bacon (1986), I, 26.

4. Cette intuition, Glanvill la partage d'ailleurs avec la plupart des membres de la Royal Society. Philippe Hamou (2001) a montré, au volume 2 de cet ouvrage magistral, comment la conscience qu'il y a dans la nature des réalités «sans pareil » motivait philosophiquement l'intérêt des empiristes anglais pour la lunette. 
La question se pose alors de savoir quel est le statut théorique de ces principes hypothétiques, et par suite, quels sont les critères qui autorisent la distinction des bonnes et des mauvaises hypothèses. Seule une analyse des facultés de l'esprit peut déterminer la nature et l'extension de notre savoir, et donc définir les conditions auxquelles les hypothèses sont recevables au titre de principes scientifiques. C'est pourquoi l'attribution à la science de son statut passe par une véritable critique des facultés.

Glanvill a donné trois versions différentes du même ouvrage : The Vanity of Dogmatizing en 1661, la Scepsis Scientifica en 1665, et enfin Against Confidence in Philosophy and Matters of Speculation en $1676^{5}$. Il s'inscrit ainsi dans le vaste mouvement de la philosophie moderne qui suspend l'élaboration du savoir à l'examen critique de ses instruments. Les raisons pour lesquelles l'âge classique peut être considéré comme l'âge de la critique sont multiples et complexes. La pluralité des régimes discursifs et l'émergence de nouveaux modèles scientifiques qui entrent en concurrence avec les savoirs autorisés par les institutions, en constituent le cadre général. Si l'on s'accorde généralement à faire de la Critique de la raison pure de Kant l'aboutissement de l'ère de la critique, il est moins facile d'en dater le début. Il semble indéniable que la subordination de la construction positive de la science à l'examen critique de la nature et des pouvoirs de l'esprit suppose l'entreprise cartésienne de mise en doute généralisée des facultés. Plus précisément la distinction cartésienne des deux substances, pensante et étendue, créait littéralement le problème de la possibilité même d'un quelconque accord entre l'idée et son objet. La nature spirituelle de toutes les idées, que l'on ne pouvait plus concevoir dans leur réalité formelle que comme des modes de l'attribut pensée (essence de l'âme) rendait difficile la compréhension de leur réalité objective comme effet des corps matériels ${ }^{6}$. Il fallait donc fonder de nouveau la validité de la relation cognitive de l'esprit à la matière. La solution cartésienne, refusant absolument tout empirisme, consistait à s'assurer, par la véracité divine, de la vérité des idées claires et distinctes, à la suite de quoi notre idée de l'essence géométrique de la matière se trouvait validée, autorisant ainsi l'élaboration d'une physique mécanique intégralement inscrite dans la géométrie. Descartes retrouvait l'idéal d'une science portant sur le réel tel qu'il est dans sa nature intrinsèque et sa nécessité. Aussi, la critique est-elle chez lui antérieure à la science, elle n'en est que le moment préalable permettant de découvrir — une fois détruits

5. Cette troisième version constitue le premier des Essays on Several Important Subjects in Philosophy and Religion, publiés en 1676. Sur les différences entre ces trois versions et leurs raisons, la littérature critique est volumineuse. Sans modifier l'essentiel de ses thèses, Glanvill change très sensiblement le style, surtout dans la dernière version. De plus en plus sobre, de plus en plus linéaire, son style répond aux exigences esthétiques de l'âge classique, qu'on les rapporte à l'influence de Descartes ou à celle des Philosophical Transactions de la Royal Society. Voir sur ce point l'étude de Sascha Talmor (1981).

6. Voyez la formulation du problème dans la troisième Méditation; cf. Descartes (1964), IX, 1, pp. 32-33. 
les préjugés des sens et de l'éducation - les fondements d'un savoir assuré de sa pertinence, pour autant que la pensée, conduite selon ses normes internes de clarté et de distinction, est assurée de porter sur un réel dont la métaphysique nous apprend qu'il est lui-même créé selon les lois que Dieu a données à notre raison. Ainsi, le réel existant, l'essence du réel, et les vérités éternelles auxquelles nous accédons, sont-ils tous de même nature, la même rationalité circulant, dans le plan des êtres créés, de l'esprit à la matière. En ce sens, Descartes a fermé la critique aussitôt qu'il l'a ouverte, et n'a jamais dépassé le schème traditionnel de la vérité comme adéquation de la chose et de l'esprit. Est-ce donc plutôt avec Locke que commence véritablement l'âge critique de la philosophie? Abandonnant le fantasme métaphysique d'un fondement du savoir, Locke s'en tient modestement à l'anatomie de l'esprit, dont l'un des résultats immédiats est de délégitimer définitivement la notion de substance, tant matérielle que spirituelle. La stricte analyse des idées a prouvé que la notion de substance, comme principe ontologique d'inhérence des attributs et des modes à leur sujet, n'était qu'une chimère scolastique. La tâche de la science s'en trouve radicalement déplacée, parce qu'elle se trouve privée de l'objet qu'elle s'était donnée depuis au moins Aristote : la connaissance de la substance. Ne pouvant plus prétendre naïvement connaître les essences réelles, elle doit s'attacher à déterminer les éléments constitutifs des essences nominales, qui ne sont rien d'autre que l'articulation des qualités sensibles reliées de façon constante en un même tout $^{7}$. On voit qu'ici la critique des facultés a des effets sur la nature même de la science en ce qu'elle lui assigne de nouveaux objets, connus selon une nouvelle manière. Or cette anatomie de l'esprit, Glanvill l'avait entreprise avant Locke, dans une orientation beaucoup plus sceptique, dont les effets sur la science elle-même devaient être encore plus profonds. Si bien qu'avec Glanvill, le rapport entre critique et science est beaucoup plus intime que ce qu'il est non seulement chez Descartes mais encore chez Locke lui-même.

Cette critique ne porte pas seulement en effet sur les mauvais usages de nos facultés, mais sur leur disproportion radicale au réel. Certes, Locke considérait que nous n'avons jamais affaire qu'aux qualités sensibles et non aux essences réelles, mais il postulait pourtant un accord entre l'ordre du réel et la cohérence des liaisons sensibles. Les différences perceptibles des essences nominales entre elles ne pouvaient guère renvoyer qu'aux différences effectives des essences réelles ${ }^{8}$. À la suite de Descartes et des cartésiens, il affirmait qu'il y a un rapport constant et réglé entre le quantitatif et le qualitatif. Pour Glanvill en revanche, la critique aboutit à la thèse d'une disparité indépassable des facultés et de l'être sur lequel elles croient porter. Que l'on envisage en effet notre connaissance des corps, notre connaissance de l'union de l'âme et du corps, ou notre connaissance de l'âme en elle-même, nous découvrons à chaque fois une opacité que la critique, loin de dissiper, ne fait qu'approfondir.

7. Voyez l'Essai III, 23, 3.

8. Essai, III, 3, 15. 
Reprenant tous les lieux communs de la critique des sens, Glanvill montre qu'ils sont étroits, trompeurs, et ne nous donnent accès qu'à la surface sensible des choses, ou plutôt même d'un tout petit nombre de choses, de sorte que nous n'avons pas même la base empirique suffisante pour élaborer des hypothèses plausibles. Il est vrai que les sens ont en eux-mêmes leurs propres correctifs, comme c'est le cas de la tour carrée que l'on voit ronde de loin, ou du bâton que l'on voit brisé dans l'eau. De fait personne n'est dupe, parce que la cohérence même de ces illusions, les exhibant comme telles, permet que nous n'y soyons pas asservis. La tour devient toujours carrée quand nous nous en approchons, le bâton retrouve toujours sa droiture quand nous le sortons de l'eau. Mais Glanvill a toujours tenu à avancer deux instances d'erreurs sensibles qu'il est impossible de corriger : le sentiment que nous avons que la terre ne se meut pas $^{9}$; l'impossibilité où nous sommes de savoir si notre perception des qualités sensibles est la même que celle des autres ${ }^{10}$. Ces deux instances ont en commun d'imposer à notre perception un monde cohérent, systématique et apparemment bien ordonné, dont nous pouvons craindre pourtant qu'il relève de la pure fiction.

Malgré son caractère massivement traditionnel, la critique des sens enveloppe une critique de la science dont il faut prendre la mesure. La science est la connaissance des causes: "science is the knowledge of things in their causes ${ }^{11}$." Or la recherche des causes est vouée à l'échec pour trois raisons principales: d'une part, nous n'avons pas accès aux ressorts intérieurs de la nature. Les éléments constitutifs des corps échappent à notre emprise. Et nous ne pouvons pas même les inférer par analogie avec ce que nous voyons de la surface des êtres. C'est en effet un fait d'expérience que les causes ne ressemblent pas à leurs effets : l'œuf ne ressemble pas à la poule, le gland ne ressemble pas au chêne. On ne peut donc supposer que les causes cachées ressemblent à leurs effets. De cette difficulté, Glanvill tirera un argument en faveur de l'usage de la lunette. Mais si la lunette nous permet de reculer les bornes de notre expérience, en approchant le lointain, elle ne permet pas de saisir la somme totale des relations causales nécessaires à la connaissance scientifique d'une seule chose. La connaissance du plus minuscule effet requiert la connaissance de l'ordre entier des causes ${ }^{12}$. Le savoir d'une seule chose présuppose donc le savoir de toute chose, parce que leurs relations mutuelles sont infinies, si bien que nous ne pouvons rien connaitre que nous n'embrassions le cercle complet du savoir ${ }^{13}$.

9. Scepsis Scientifica, chapitre 11, pp. 57-63.

10. Ibid., chapitre 26 .

11. Essays, I, p. 14.

12. Scepsis Scientifica, p. 159: «thus then, to the knowledge of the most contemptible effect in nature, 'tis necessary to know the whole syntax of causes".

13. Essays I, p. 16: "Everything in nature hath relation to divers others, so that no one being can be perfectly known without the knowledge of many more. Yea, everything almost hath relation to all things; and therefore he that talk of strict science pretends to a kind of omniscience. [...] So that each science borrows from all the rest, and we attain not any single one without comprehending the whole circle of knowledge. " 
Enfin, et surtout, la critique des sens a conduit Glanvill à une troisième critique de la causalité dont on a souvent noté la proximité avec celle de Hume. Plutôt que de chercher si Glanvill a ou non influencé Hume, il convient de se demander si leur scepticisme devait les conduire à poser le problème de la causalité comme ils le font. "Ce n'est pas par l'intuition simple mais par la déduction et l'inférence que nous connaissons les causes ${ }^{14}$. " Cette inférence usuelle, nous la faisons sur l'expérience d'une simple concomitance : il se trouve que nous sentons toujours de la chaleur quand nous nous approchons du feu, que nous percevons toujours de la lumière quand nous voyons le soleil. Comment passe-t-on de la concomitance constamment perçue entre le feu et la chaleur à l'affirmation d'une relation de causalité non perçue ? La réponse de Glanvill - implicite bien que claire - est que cette opération indue se fait par la seule répétition. La répétition de la concomitance nous la fait traduire immédiatement en causalité, et l'on peut même aller jusqu'à dire que Glanvill " théorise » la relation causale en termes de croyance, comme il apparaît dans l'exemple qu'il donne. Une simple expérience de pensée suffit en effet à établir que l'inférence causale ne procède d'aucun fondement rationnel, et la renvoie ainsi à sa nature brute de certitude de fait, c'est-à-dire de croyance : « $\mathrm{Si}$ nous n'avions jamais perçu le soleil ou les étoiles que sous un ciel nuageux, et si le jour s'était toujours levé au moment où se lève le vent, et n'avait jamais baissé qu'en même temps que le vent baisse, nous croirions fermement [we should have believed firmly] que l'un est la cause de l'autre ${ }^{15}$. » Glanvill dit ici tout autre chose que ce que disait la traditionnelle mise en garde scolastique contre le sophisme post hoc ergo propter hoc. Loin de se contenter de dénoncer un mauvais usage du raisonnement causal, il y réduit notre connaissance habituelle des causes. Il formule donc, mais en creux, l'hypothèse que l'inférence causale n'est au fond qu'une croyance qui repose sur la confiance arbitraire que nous faisons à l'habitude ${ }^{16}$. En ce sens il pose le problème auquel Hume répondra, car Hume ne réduit pas la causalité à la concomitance et ne se contente pas d'exhiber la part de l'habitude dans l'inférence. Il affirme que l'habitude, en liant l'idée de la cause à la perception de l'effet, produit une détermination subjective à passer de l'une à l'autre, détermination qui fait le fond de l'inférence. En d'autres termes, là où Glanvill dénonce une illusion, Hume met au jour une dynamique anthropologique, là où Glanvill désigne un préjugé, Hume construit une théorie positive de la croyance.

14. Against Confidence in Philosophy and Matters of Speculations, Essays, I, p. 14. Voyez le même développement dans la Scepsis le chapitre 23, et notamment les pages 142sq.

15. Ibid.

16. Sur Glanvill et Hume, voir Popkin (2003). L'interprétation de Sascha Talmor (1981, p. 64 sq.), selon laquelle les ressemblances entre Glanvill et Hume sur ce point sont superficielles, puisque Glanvill est un penseur de la contingence alors que Hume est un penseur de la nécessité (et quoi qu'on pense de cette interprétation de la causalité selon Hume) n'invalide nullement l'essentiel, à savoir que l'inférence causale est, chez l'un comme chez l'autre, une croyance. 
Outre ces difficultés, qui ne sont au vrai que des difficultés de fait que la science moderne pourrait surmonter, en utilisant par exemple les microscopes et les télescopes, de manière à nous rapprocher de la vue qu'Adam pouvait avoir des choses ${ }^{17}$, il en est une de droit, qui interdit à jamais de penser que la science puisse dire le réel. L'essentiel reste en effet que nous ne pouvons jamais nous assurer que ce qui est principe pour nous est principe de l'être. L'affirmation sceptique qu'enveloppe la critique de la raison est que le réel excède notre raison: "what seems impossible to us may not be so in nature ${ }^{18}$ ". Les lois de la logique sont donc ontologiquement muettes, parce que l'expérience est la contingence même. Pour tout ce qui relève des matters of fact en effet, il est impossible de démontrer qu'ils ne peuvent être autrement qu'ils ne sont, puisque cela n'implique pas de contradiction. Si je ne peux concevoir un cercle carré, je peux concevoir sans aucune contradiction que le feu ne brûlera pas ma main qui s'en approche ou que l'eau ne se transformera pas en glace sous $0{ }^{\circ} \mathrm{C}$, ce qui suffit pour que la chaîne des causes reste indéfiniment ouverte. La cause n'indique pas la nécessité de son effet. Cette conception de l'expérience comme contingence, que Glanvill est le premier à dramatiser avec tellement d'emphase, devait avoir des conséquences incalculables. Non seulement Glanvill, en définissant l'expérience par la contingence, devance Hume, mais, si l'on y prend garde, on remarquera que cette définition de l'expérience par la contingence est le motif capital qui a conduit les savants à substituer à la recherche des causes la détermination des lois. Le fait que nous ne puissions remonter de la contingence à la nécessité par la seule considération des effets contraint le regard scientifique à s'en tenir au repérage et à la mesure des régularités phénoménales, de manière à déterminer le rapport constant des phénomènes, formulable mathématiquement ${ }^{19}$.

On comprend alors le sens profond du recours à l'expérience, entendue comme experientia et comme experimentum, comme retour au sensible en même temps que mise à la question du sensible par l'œil aidé de ses instruments. Car puisque l'expérience est la contingence même, le pays des possibles que les rationalistes placent en Dieu se trouve désormais situé dans le réel, à portée du regard

17. Sur l'importance de la figure de l'Adam prélapsaire, dont Glanvill trace le portrait dans les premiers chapitres de la Scepsis, comme paradigme de la science instrumentalisée que met en œuvre la Royal Society, voir les très belles analyses de P. Hamou (2001), notamment le chapitre 3.

18. Scepsis Scientifica, p. 152. Voyez aussi dans le premier des Essays, p. 15 : «our best natural knowledge is imperfect in that after all our confidence, things still are possible to be otherwise ». À cet argument, Glanvill joint un argument tiré de la toute-puissance divine : «for to say, the principles of nature must needs be such as our philosophy makes them, is to set bounds to omnipotence, and to confine infinite power and wisdom to our shallow models ", id., p. 156. Cette formulation rappelle les analyses célèbres de Montaigne sur la toute-puissance divine: «Nous lui prescrivons des bornes, nous tenons sa puissance assiégée par nos raisons» (II, 12, p. 523).

19. On sait que Popkin considérait ce tournant comme la caractéristique essentielle de la modernité, qu'il définit par la réponse qu'elle a su donner ainsi à la «crise pyrrhonienne ». Voyez sa théorie du scepticisme constructif, dans Popkin (2003). Chez Glanvill, ce tournant se fait sur la question de la contingence. C'est pourquoi son œuvre intéresse l'histoire de la métaphysique. 
savant. En plaçant l'univers des possibles dans le monde des phénomènes, l'empirisme « radical » renverse l'ontologie. L'ordre des possibles n'est plus identique à l'ordre des essences, dont l'existence effective n'est que l'actualisation, inévitablement restreinte - certains possibles ne se réalisant pas. Le monde de l'expérience est désormais coextensif au monde des possibles. Dès lors, l'expérience scientifique a pour tâche de faire reculer les bornes du connu, en allant chercher des réalités sans pareilles qui contraignent la théorie à réviser incessamment ses lois. L'expérience n'est plus le lieu de l'application des lois de la nature, elle est le lieu de la mise en cause de ces lois par la recherche de réalités qui brisent le cadre de ce qui jusqu'ici était tenu pour possible. C'est la raison profonde de l'engouement pour le télescope et le microscope, qui n'approchent pas seulement ce qui est lointain ou petit mais qui changent la nature même du visible.

Ce n'est pas seulement dans leur rapport au monde que les facultés sont privées d'un fondement capable d'en garantir le bon usage, c'est aussi notre âme dans son rapport à elle-même. Nous sommes aussi étrangers à nous-mêmes qu'aux Américains ${ }^{20}$. Il n'y a pas chez Glanvill de cogito.

S'il fallait s'attendre à trouver la certitude quelque part, on peut penser que ce dût être dans la connaissance de l'âme, qui est en effet notre moi, et dont les sentiments nous sont intimement connus. Pour ce qui est des choses extérieures, il n'est pas étonnant que nous les ignorions, puisque nous ne pouvons descendre jusqu'aux éléments secrets de la nature ni voir les premiers ressorts et les rouages qui mettent l'ensemble en mouvement. Nous ne voyons que de petites parcelles de la structure totale et nous manquons de phénomènes pour faire des hypothèses complètes et sûres. Mais si ce par quoi nous connaissons le reste des choses ne se connaît pas lui-même, si notre âme est étrangère à ce qu'il y a en elle-même, alors même qu'elle jouit à cet égard de beaucoup plus d'avantages que pour la connaissance des faits de la nature extérieure, je pense qu'on sera contraint d'avouer ici cette ignorance que je voudrais qu'on admitt ${ }^{21}$.

S'inspirant sans doute des critiques de Montaigne dans l' " Apologie de Raimond Sebond $»^{22}$, Glanvill accumule les définitions de l'âme pour en exhiber la variété et le ridicule ${ }^{23}$. Comme chez Montaigne, le catalogue produit un effet d'isosthénie, et même d'asthénie, la masse des définitions ayant pour effet de les effacer toutes plutôt que de les neutraliser les unes par les autres. La seule chose dont nous soyons assurés, c'est que les propriétés de l'âme sont radicalement hétérogènes à celles du corps. L'âme est une substance spirituelle, de la nature de laquelle nous ignorons tout. Mais cette seule connaissance de la spiritualité de l'âme déplace le problème, car si l'âme et le corps sont deux substances hétérogènes, leur union devient absolument inintelligible ${ }^{24}$.

20. Scepsis Scientifica, Address, p. 5.

21. Scepsis Scientifica, p. 13 (traduction nôtre).

22. Voyez II, 12, p. 546sq.

23. Scepsis Scientifica, pp. 13-15

24. Scepsis Scientifica, p. 15 : «That we are a compound of beings distant in extreams, is as clear as noon. But how the purer spirit is united to this clod is a knot too hard for our degraded intellects to unity. " 
$\mathrm{Au}$ vu de cette critique, il semblerait que toute science soit définitivement invalidée, et que Glanvill, comme les sceptiques anciens, adhère à l'idée selon laquelle «il n'est science de rien ». Tel n'est pourtant pas le cas. Glanvill conçoit son œuvre comme une participation à la promotion du savoir, et la critique se joint toujours chez lui à l'éloge de la science moderne ${ }^{25}$. L'ensemble de ses livres forme comme un immense diptyque où l'assurance des progrès scientifiques fait pendant à la ruine de leurs fondements métaphysiques. Aussi prend-il soin de démarquer son scepticisme de celui des anciens et renverse-t-il l'objection de Thomas White, qui l'avait accusé de détruire la science. $\mathrm{Si}$ « les dogmatiques sont les seuls sceptiques ${ }^{26}{ }^{2}$, c'est que, faisant confiance à l'usage immédiat, non critique, de leurs facultés, ils ne peuvent guère que redoubler l'ignorance spontanée en la masquant sous la profusion d'un discours verbal qui résout tout en renvoyant aux qualités occultes et aux pouvoirs secrets de la nature. Dans l'essai Of Scepticism and Certainty, Glanvill renvoie dos à dos les dogmatiques et les sceptiques anciens. Les pyrrhoniens comme les académiciens doutaient pour douter, leur grand principe étant qu'aucune vérité ne pouvait être découverte ${ }^{27}$. Les sceptiques anciens, si tant est qu'on puisse accorder foi aux témoignages qui nous retracent leurs vies et leurs doctrines, étaient des fous, à moins qu'ils ne fussent des provocateurs. Quant au scepticisme moderne, il n'a de sens que de désigner les penseurs qui savent que leurs principes ne sont que des hypothèses ${ }^{28}$.

Il ne faudrait pourtant pas croire que le scepticisme selon Glanvill soit moins radical que le scepticisme ancien, au motif qu'il se rassemble et se résume tout entier dans la réduction du principe à l'hypothèse. Car cette réduction même suppose un déplacement de la question de la vérité sur le plan du droit, que les sceptiques anciens avaient nécessairement ignorée. Les sceptiques anciens se contentaient en effet de nier le fait que nous ayons accès à la science - si l'on entend par science la connaissance du réel en tant que tel. Glanvill, pour sa part, hérite de l'argument cartésien du Dieu trompeur, qui contraint la théorie de la connaissance à se situer sur un tout autre plan. Ayant parfaitement conscience de la gravité de l'argument du Dieu trompeur et de ses

25. En 1668, il publie un ouvrage dont le titre même est éminemment baconien : Plus Ultra: or the Progress and Advancement of Knowledge since the Days of Aristotle; le troisième des Essays de 1676 porte sur les Modern Improvements of Useful Knowledge.

26. "The dogmatists are the only scepticks », Scepsis scientifica, p. 127.

27. "And indeed their doubting and suspension was not in order to the forming a surer judgment, but a resolution to sit down forever in despair of knowledge: And therefore they were very improperly called zetetikoi, seekers, since their great principle was that nothing was to be found. Upon the whole, it was not without cause that Cicero, Aristocles, and other sober philosophers spoke of their way as down-right madness ", Of Scepticism and Certainty, p. 42.

28. Les sceptiques modernes "propose their opinions as hypotheses that may probably be the true accounts, without peremptorily affirming that they are. This, among others, hath been the way of those great men, the Lord Bacon and Descartes, and is now the method of the Royal Society of London, whose Motto is Nullius in verba. This is scepticism with some; and if it be so, "tis such scepticism as is the only way to sure and grounded knowledge ", ibid., p. 44. 
conséquences définitivement dévastatrices ${ }^{29}$, Glanvill retient l'argument sceptique tout en rejetant la solution cartésienne. Nous ne pouvons pas savoir si nous ne sommes pas faits de manière à nous tromper toujours, même en ce qui concerne nos idées les plus claires et les plus distinctes. Descartes avait cru pouvoir invalider l'argument en démontrant que Dieu ne peut pas être trompeur. Mais à la suite d'Arnauld ${ }^{30}$, Glanvill considère que la soi-disant preuve de Descartes est circulaire: "Je pense que Descartes est infidèle à sa méthode quand, de l'idée de Dieu, il s'efforce de prouver que Dieu existe et, de son existence, que nos facultés sont fiables, car la fiabilité de nos facultés est présupposée pour prouver l'existence de Dieu. [...] De sorte que ce grand homme semble raisonner dans un cercle ${ }^{31}$. "Or c'est précisément parce que la preuve de Descartes ne prouve rien que nous restons sous la possibilité métaphysique du Dieu trompeur: "il n'est peut-être pas absolument impossible que nos facultés soient constituées de telle sorte qu'elles nous trompent toujours concernant les choses que nous jugeons les plus certaines et assurées ${ }^{32} »$. Dès lors, il est nécessaire de distinguer deux types de certitude : une certitude fondée métaphysiquement, que Glanvill appelle "certitude infaillible » (infallible certainty), et une certitude " psychologique ", qu'il nomme certitude indubitable (indubitable certainty) ${ }^{33}$. La certitude infallible est l'assurance absolue (absolute assurance) que les choses sont comme nous les concevons. Elle implique que nous puissions dire pourquoi les choses sont comme elles sont et non autrement. De cette certitude métaphysique nous sommes privés. Mais nous pouvons atteindre la certitude indubitable, qui se définit comme le ferme assentiment que nous donnons à ce qui ne présente aucune raison de douter ${ }^{34}$. Ce qui est indubitable de fait suffit pour la construction de la vérité. Il semble d'abord que Glanvill, se satisfaisant, faute de mieux, de critères purement psychologiques, opère une régression par rapport à la hauteur à laquelle Descartes avait hissé le problème des critères de vérité. En affirmant que nous pouvons faire confiance à ce qui n'est pas douteux, il subjectivise la théorie de la connaissance, alors que Descartes semblait avoir posé dans les Méditations métaphysiques une question autrement radicale, une question de droit : de quel droit tiens-je ce qui est subjectivement indubitable pour vrai ? Ainsi posée, la théorie de la

29. Richard Popkin (2003) a magistralement montré comment Descartes, voulant surmonter la «crise pyrrhonienne » qui dynamise selon lui toute la philosophie de l'âge classique, a engendré un scepticisme redoutable par l'argument du Dieu trompeur, conduisant par son échec ses successeurs à renoncer au projet d'un savoir fondé métaphysiquement pour se contenter de critères relatifs, probabilistes et expérimentaux, qui dessinent la figure de la science moderne. Cette dernière édition comporte un chapitre sur Glanvill à la fin duquel Popkin considère que la science, telle que Glanvill et les hommes de la Royal Society en formulent le programme, est, mutatis mutandis, celle que nous pratiquons encore aujourd'hui (p. 218).

30. Voyez Descartes (1964), IX, 1, p. 166.

31. Essays, II, Of Scepticism and Certainty, p. 48.

32. Ibid., p. 50.

33. Ibid., p. 47.

34. Ibid.: "A firm assent to anything of which there is no reason of doubt ». 
connaissance exigeait une investigation métaphysique qui seule donne sa véritable ampleur à l'orientation critique de la philosophie où se joue sa modernité. Mais en vérité, loin que Glanvill ait fermé l'orientation critique en abaissant l'exigence des critères de vérité à leur dimension psychologique, c'est dans $O f$ Scepticism and Certainty que l'on trouve la formulation la plus claire de l'investigation de droit. Car Descartes aurait effectivement posé la question du droit que nous avons de tenir ce qui est subjectivement indubitable pour une vérité objective s'il avait douté de l'évidence. Or rien, dans le texte des Méditations, n'autorise une telle interprétation, et la vulgate qui distingue dans la première Meditatio un doute méthodique qui cherche l'évidence d'un doute métaphysique qui porte sur l'évidence elle-même ne résiste pas à la lecture. Si l'on doute en effet des mathématiques, si l'on en vient à envisager l'hypothèse d'une folie constitutive de l'esprit, il y a des raisons pour ce faire qui, pour être légères et métaphysiques, n'en sont pas moins de vraies raisons. De fait, les mathématiciens commettent des paralogismes. De fait, l'hypothèse d'un Dieu trompeur constitue une très réelle raison de douter, car dès lors que l'on prend toute la mesure de ce qu'enveloppe l'attribut de la toute-puissance divine, il est indéniable qu'il peut tromper. Bien qu'elle ne soit qu'une vetus opinio, l'existence d'un Dieu tout-puissant n'implique pas contradiction, de sorte qu'il est réellement possible qu'il existe un Dieu tout-puissant qui, en tant que tel, peut donc me tromper touchant les vérités les plus claires. C'est là une raison de douter, dont l'effet immédiat est d'effacer l'évidence de nos idées claires et distinctes. Aussi, dès lors que l'analyse intrinsèque de l'idée d'un Dieu en effet tout-puissant aura montré que la tromperie ne peut être qu'une marque d'impuissance, il n'y aura plus aucune raison de douter de la véracité de Dieu. Et cette absence de raison de douter suffira seule à en faire un Dieu vérace. Descartes, n'ayant jamais douté de l'évidence, n'est donc jamais sorti de l'ordre du fait. Il a seulement cherché une évidence absolument transparente. En revanche Glanvill, parce qu'il ne donne pas son assentiment à la preuve cartésienne de l'existence de Dieu, crée littéralement la scission du fait et du droit, sous la forme de la différence jamais surmontée entre la certitude infaillible et la certitude indubitable. Nous restons soumis à la possibilité métaphysique d'un Dieu trompeur; il est toujours possible, en droit, que toute notre fabrique psychique soit folle. Il se trouve simplement que nous n'avons aucune raison de le supposer, mais la possibilité métaphysique de l'erreur absolue reste ouverte, alors que non seulement elle ne l'est pas mais ne l'a jamais été chez Descartes. C'est cette possibilité maintenue qui motive la distinction des deux types de certitude, dont la signification historique est capitale. En instaurant la question du droit, à laquelle Glanvill répond par la négative (nous n'avons pas le droit de prétendre à l'infaillibilité), il met au jour le fait que la confiance que nous faisons à la raison relève de la croyance. Dans une page remarquable par sa lucidité métaphysique, Glanvill réécrit la métaphysique de Descartes en la renversant de fond en comble: 
Je ne peux douter sans penser, même si rien [de ce que je pense] n'est tel que je le pense; et je ne puis douter que moi, qui pense, je suis. Je suis certain d'avoir des idées et des conceptions de choses à l'extérieur de moi, comme celles de Dieu, du ciel, de la terre, etc. Jusqu'ici, ce philosophe est en sécurité, et notre assentiment est complet. Il en va de même pour ces autres choses, à savoir que nous pouvons composer ou disjoindre ces images par affirmation ou négation, que nous avons une faculté de raisonner et d'inférer une chose à partir de l'autre. Tout ceci, nous le percevons clairement et le sentons en nous-mêmes, quelque incertitude qu'il y ait quant aux autres sujets. À ceci, nous donnons un assentiment ferme et résolu, et n'avons pas la moindre raison d'en douter. Outre ces principes, il s'en trouve d'autres, plus généraux, dans notre esprit, dont nous présupposons la valeur et que nous utilisons dans tous les raisonnements et toutes les affirmations auxquels nous donnons un plein assentiment, comme par exemple que chaque chose est ou n'est pas, qu'une chose ne peut pas être et ne pas être sous les mêmes rapports, que rien n'est sans attribut, que ce que nous concevons appartenir ou ne pas appartenir à une chose, nous pouvons l'affirmer ou le nier de cette chose.

Tels sont les principes de toutes les propositions et de toutes les ratiocinations, quelles qu'elles soient; et nous y assentissons pleinement dès que nous en comprenons le sens. J'y ajoute ce grand principe : nos facultés sont fiables, c'est-àdire que ce que notre entendement dit des choses que nous concevons clairement et distinctement est vrai et convient avec la réalité des choses elles-mêmes. C'est un principe auquel nous croyons fermement, mais que nous ne pouvons prouver [this is a principle that we believe firmly, but cannot prove], car toute preuve et tout raisonnement le suppose ${ }^{35}$.

Ainsi donc la certitude que notre science convient avec le réel n'est que croyance. C'est dans le langage même de Descartes que Glanvill montre, contre Descartes, que la raison ne saurait sans pétition de principe se fonder elle-même : on ne déduira pas infailliblement de la raison la preuve que Dieu garantit la certitude infaillible de la raison. En d'autres termes, la certitude indubitable qu'offre l'évidence scientifique n'est pas ordonnée à la certitude infaillible qui n’appartient qu'à Dieu. La science se trouve ainsi déliée de son ancrage métaphysique. Au plus profond de la pensée rationnelle gît la croyance, c'est-à-dire l'affirmation que, si nous pouvons faire confiance, nous devons pourtant faire seulement confiance à nos facultés, lorsqu'elles sont correctement exercées.

Nous ne sommes pas pour autant renvoyés au pur arbitraire d'une croyance de fait. La certitude indubitable, sans jamais pouvoir devenir infaillible, est en effet fondée sur la connaissance que nous avons de Dieu. Nous savons que Dieu existe, et qu'il n'est pas trompeur. On pourrait croire qu'il s'agit là d'un retour subreptice à Descartes, et que Glanvill se contredit quand il rejette la preuve cartésienne de l'existence de Dieu, tout en invoquant la véracité divine: "Nous savons que les premiers principes sont certains et que nos sens ne nous trompent pas, parce que Dieu, qui nous les a donnés, est vérace 
et bon $^{36}$.» Pourtant, si la fonction de Dieu est la même chez Descartes et Glanvill, la manière dont nous le connaissons n'est pas la même chez l'apologète anglican et chez le philosophe français. Alors que Descartes dans ses Méditations Métaphysiques parvenait à Dieu sans se référer jamais à la religion, la preuve de l'existence de Dieu chez Glanvill repose sur une analyse de la religion plus que de la théologie naturelle. À cet égard, son texte le plus important est peut-être le cinquième des Essays, The Agreement of Reason and Religion, dont le propos, évidemment apologétique, consiste à montrer, contre les athées et les enthousiastes, que la raison et la foi concourent ensemble au service de l'humanité ${ }^{37}$. L'approche est profondément rationaliste: «Reason lays the very corner stone of religion ${ }^{38}$. » La foi repose en effet sur la raison seule puisque nous ne pouvons admettre la divinité de l'Écriture que si nous savons d'abord qu'il existe un Dieu et qu'il est providentiel. Mais la seule preuve recevable de l'existence de Dieu est la preuve cosmologique:

La raison prouve l'existence de Dieu par la beauté, l'ordre, la finalité et l'utilité pour les créatures. Ce sont là les arguments démonstratifs en faveur de l'existence d'un Esprit [Mind] sage et omnipotent, qui a organisé [framed] toutes choses avec une régularité et une exactitude parfaites ${ }^{39}$.

Le fameux argument du Design n'est plus seulement l'un des arguments de l'appareil démonstratif, il est le seul. C'est donc l'expérience qui est le moteur unique de la preuve. Mais à cette preuve, il en faut joindre une seconde, d'ordre proprement religieux. Une fois que nous savons par la considération de l'ordre cosmique que Dieu existe et qu'il est providentiel, il nous faut nous assurer que l'Écriture sainte est bien sa parole. Glanvill reprend ici l'argumentaire rationaliste de l'apologétique traditionnelle, tel qu'on le trouve élaboré traditionnellement dans l'Église catholique. La divinité de l'Écriture est établie par la concordance des témoignages. Le principe en est qu'il est moralement impossible que tant d'hommes se soient accordés pour mentir, ou aient pu être victimes des mêmes illusions :

Que le Christ et ses apôtres aient fait ce que les Livres rapportent est affaire de témoignage, et la raison éclaire la validité du fait par le concours des circonstances très nombreuses qui montrent que ceux qui les relatèrent d'abord ne pouvaient se tromper eux-mêmes et ne voulaient nous tromper, pas plus qu'ils ne l'auraient pu s'ils l'avaient voulu ${ }^{40}$.

Nous ne renonçons pas plus à notre raison quand nous croyons que l'Écriture nous révèle la parole de Dieu que quand nous croyons que Jules César

36. The Agreement of Reason and Religion, Essays, V, p. 20.

37. Sur l'apologétique de Glanvill, voyez J. I. Cope (1956).

38. Ibid., p. 7.

39. Ibid.

40. Ibid., p. 8. 
fut tué aux ides de mars $44^{41}$. Pas plus qu'il ne serait pas raisonnable de douter de la vie et de la mort de César, il n'est raisonnable de douter de la vie et de la mort du Christ, l'une et l'autre ayant très exactement le même titre à notre assentiment.

Cette manière de démontrer l'existence de Dieu par la contemplation de l'ordre naturel et par la considération de la divinité de l'Écriture est essentielle. Elle permet à Glanvill de retrouver Dieu en échappant au cercle de la métaphysique cartésienne, puisque les preuves sont ici expérimentales, donc dotées d'une validité scientifique. Elle permet aussi, par le jeu de la confirmation réciproque de l'Écriture par l'expérience et de l'expérience par l'Écriture, de comprendre que la circularité entre raison et foi n'est pas une circularité vicieuse. Glanvill peut donc à la fois maintenir que la raison est un objet de croyance et que la croyance est raisonnable: "The belief of our reason is an exercise of faith, and faith is an act of reason ${ }^{42}$. " On peut comprendre désormais pourquoi la «théologie » échappe au scepticisme. Elle est fondée sur l'expérience, expérience de l'ordre du monde, expérience de la divinité de l'Écriture.

Si l'œuvre de Glanvill témoigne d'une mutation dans les rapports que la science entretient avec la vérité, c'est en ce sens que son scepticisme le conduit à mettre au jour le fond de croyance qui anime la démarche rationnelle. La raison n'est pas et ne peut pas être à elle-même le principe de sa propre fondation. Nous devons y croire, d'une croyance qui est «an exercise of faith». Mais cette croyance est naturelle: les principes de la raison «sont ce que l'instinct est pour les autres créatures ${ }^{43}$ ". Glanvill naturalise l'exercice de la raison, ce qui ne contredit nullement le fait qu'il conçoive la science comme un ensemble discursif intégralement construit sur des hypothèses. Pas plus que chez Hume, naturalisme et constructivisme ne s'entrechoquent. C'est que les hypothèses auxquelles parvient le savant sont toujours en droit soumises à révision.

41. C'est ce type d'argument que Glanvill développe pour défendre sa théorie de l'existence des sorciers. Sur le complexe juridique, scientifique, théologique et politique de la sorcellerie dans l'Angleterre du XVII ${ }^{e}$ siècle, voir les analyses de Barbara Shapiro (1983), notamment le chapitre 6. Sur Glanvill, voyez les pages 213 sq.: "Glanvill attacked his opponents on methodological grounds. [...] The natural scientist, after all, did not deny facts simply because he had no explanation for them" (p. 214).

42. Ibid., p. 21. On voit qu'il faut nuancer le propos de H. Van Leeuwen (1970), qui faisait de Wilkins et de Glanvill les figures de la sécularisation du problème de la certitude dans la pensée anglaise du XVII e siècle. Il est indéniable que Glanvill applique les critères de l'adhésion religieuse à la science. Mais il est tout aussi indéniable que le dernier mot de sa théorie de la connaissance, tel qu'il est développé dans l'Essay $\mathrm{V}$, noue de manière très rigoureuse la croyance enveloppée dans la religion et l'assurance scientifique. D'un certain point de vue donc, Glanvill freine la sécularisation, en détruisant la preuve métaphysique de l'existence de Dieu. Mais d'un autre point de vue, sa lucidité quant à la pétition de principe qu'enveloppe la preuve métaphysique le conduit à comprendre que la raison elle-même n'est en son fond qu'une croyance. On ne peut qu'être frappé de l'homologie de cette démarche avec celle de Hume, dont la critique entreprise dans les Dialogues sur la religion naturelle et l'histoire naturelle de la religion dénouera définitivement croyance et théologie.

43. Ibid., p. 6. 
L'expérience fera seule le partage des hypothèses. Non seulement l'expérimentation de laboratoire, mais aussi et surtout les fruits de l'expérience. Les ouvrages de Glanvill qui manifestent son enthousiasme scientifique ne sont pas seulement des pamphlets de circonstance dus à son militantisme en faveur de la Royal Society. Ils sont aussi l'expression d'une théorie de la connaissance qui n'a d'autre critère que les résultats. On mesure la validité d'une théorie à sa fécondité technique. Si l'on arrivait, grâce à l'hypothèse mécanique, à inventer le téléphone $e^{44}$, on aurait de bonnes raisons de croire que le mécanisme est une grille correcte de lecture du monde. La maîtrise technique du monde et du corps n'a donc pas le même statut que chez Descartes, pour qui elle est la conséquence des principes. C'est en effet selon Descartes parce que les principes de la philosophie sont vrais que nous pourrons fabriquer un univers où la technique améliore la condition humaine. Chez Glanvill à l'inverse, c'est parce que la maîtrise est effectuée que l'on peut s'assurer que les principes étaient bons. L'efficacité technique que permet une théorie est l'indice de sa valeur. Cela sans affirmation ontologique, c'est-à-dire sans que l'on dépasse le stade des hypothèses. Ce n'est pas parce que les principes sont vrais qu'on peut maîtriser la nature, c'est parce qu'on maîtrise la nature qu'il apparaît que les principes étaient, non pas même vrais, mais bons. Le constructivisme de Glanvill ne va pas sans un certain pragmatisme avant la lettre, en ce sens que la science se fait toujours au futur antérieur: au vu des réalisations techniques rendues possibles par telle théorie, on aura eu raison de faire confiance à la théorie. On est proche ici de Bacon, si proche même que les membres de la Royal Society ont pu croire de bonne foi être baconiens, et voir dans leur institution la réalisation approchée de la Nouvelle Atlantide. Mais il y a encore chez Bacon un fond de réalisme qui disparaît avec Glanvill. Bacon prétend que la connaissance des lettres de l'alphabet de la nature peuvent être connues, de manière que nous puissions former dans l'entendement le modèle vrai de la nature ${ }^{45}$. Au-delà de toutes leurs différences, qu'il ne s'agit évidemment pas de minorer, Bacon et Descartes partagent une même conception de la maîtrise technique du monde: c'est parce que la rationalité du monde est intrinsèquement technique que sa maîtrise est possible. Avec Glanvill s'ouvre un tout autre univers épistémologique. La maîtrise technique n'est que le signe que la science se construit dans le bon sens. Elle ne signifie pas que le réel obéit de fait à une rationalité technique ni qu'il y ait un accord fondamental entre la raison et le réel. C'est bien plutôt parce que dans sa richesse et son étrangeté radicales, le monde déborde l'emprise rationnelle que rien, jamais, ne pourra exempter la science de sa confrontation continue avec l'expérience.

44. Voyez, pour le «téléphone », la Scepsis p. 149sq.

45. Bacon (1986), II, 8, pp. 193-194. 


\section{Bibliographie:}

Bacon, F. Novum Organum, Paris, Presses Universitaires de France, 1986.

Cope, J. I. Joseph Glanvill: Anglican Apologist, St. Louis, Washington University Press, 1956.

Descartes, R. Euvres de Descartes, Paris, Vrin, 1964-1974, 11 vol.

Glanvill, J. Collected Works, Georg Olms Verlag, Hildesheim-New York, 1970sq.

Hamou, P. La mutation du visible, Lille, Presse du Septentrion, 2001, 2 vol.

Montaigne, M. Les Essais, Paris, Presses Universitaires de France, 2004.

Popkin, R. H. The History of Scepticism from Savonarola to Bayle, Oxford, Oxford University Press, 2003.

—. "Joseph Glanvill: A Precursor of David Hume ", The Journal of the History of Ideas, 14, 1953, p. 292-303.

Shapiro, B. Probability and Certainty in $17^{\text {th }}$ England, Princeton, Princeton University Press, 1983.

Talmor, S. Glanvill: the Uses and Abuses of Scepticism, Oxford-New York, Pergamon Press, 1981.

Van Leeuwen, H. G. The Problem of Certainty in English Thought. 1630-1680, La Haye, Martinus Nijhoff, 1970. 